\title{
EVIDENCE FOR A PSEUDOKNOT IN THE 3' UNTRANSLATED REGION OF THE BOVINE CORONAVIRUS GENOME
}

\author{
G. D. Williams, ${ }^{1}$ R. -Y. Chang, ${ }^{2}$ and D. A. Brian ${ }^{2}$ \\ ${ }^{1}$ Program in Biotechnology \\ ${ }^{2}$ Department of Microbiology \\ University of Tennessee \\ Knoxville, Tennessee 37996-0845
}

\begin{abstract}
A potential pseudoknot was found in the $3^{\prime}$ untranslated region of the bovine coronavirus genome beginning 63 nt downstream from the stop codon of the $\mathrm{N}$ gene. Mutation analysis of the pseudoknot in a cloned defective interfering RNA indicated that this structural element is necessary for defective interfering RNA replication.
\end{abstract}

\section{INTRODUCTION}

The 291 nt 3' untranslated region (UTR) of the bovine coronavirus (BCV) likely contains sequences that serve as a promoter for minus-strand synthesis during RNA replication. To explore the structural features that might be important for this function, a computer program was used to predict thermodynamically stable secondary structural elements. This analysis identified a potential pseudoknot beginning $63 \mathrm{nt}$ downstream from the termination codon of the $\mathrm{N}$ gene having two stems with a high negative free energy. A phylogenetic comparison with other coronaviruses indicated conservation at the secondary and tertiary levels.

To investigate the functional significance of the proposed pseudoknot in viral RNA replication, site-directed mutagenesis of a cloned $2.2 \mathrm{~kb}$ reporter containing defective interfering RNA (pDrepl) was undertaken to disrupt and restore base pairing in the first stem of the pseudoknot. Mutants were then tested for replication. These experiments strongly suggest a role for the pseudoknotted structure in viral RNA synthesis.

\section{MATERIALS AND METHODS}

Cloning and construction of the bovine coronavirus defective-interfering RNA which carries a reporter sequence of $30 \mathrm{nt}$ (pDrep1) have been described ${ }^{1}$. Synthesis of pDrep1 
RNA transcripts and assay for pDrep 1 replication using Northern analysis were done as previously described ${ }^{1}$. Site-directed mutations of pDrep1 3' UTR were done with a previously published PCR-based mutagenesis procedure ${ }^{2}$. For these, oligodeoxynucleotides UCUAAAC and AGAUUUG were used. For computer analysis, the Microgenie program (Beckman Instruments) which employs the Tinoco algorithm for analysis of RNA secondary structure $^{3}$ was used.

\section{RESULTS}

A computer-assisted analysis of RNA secondary structures in the BCV 3' UTR showed the existence of two stem regions between nucleotides -173 and -226 from the base of the poly(A) tail. The downstream stem, stem 2, has a predicted stability of $-10.2 \mathrm{kcal} / \mathrm{mole}$, and the upstream stem, stem 1, which involves base-pairing between upstream sequences and the loop of stem 2 , has a predicted stability of $-8.6 \mathrm{kcal} / \mathrm{mole}$ (Fig. 1). The potential structure formed would be considered an H(hairpin)-type pseudoknot in which stems 1 and 2 of the folded structure would become adjacent to each other to cause coaxial stacking of the two stems and formation of a quasi-continuous double helix ${ }^{4}$.
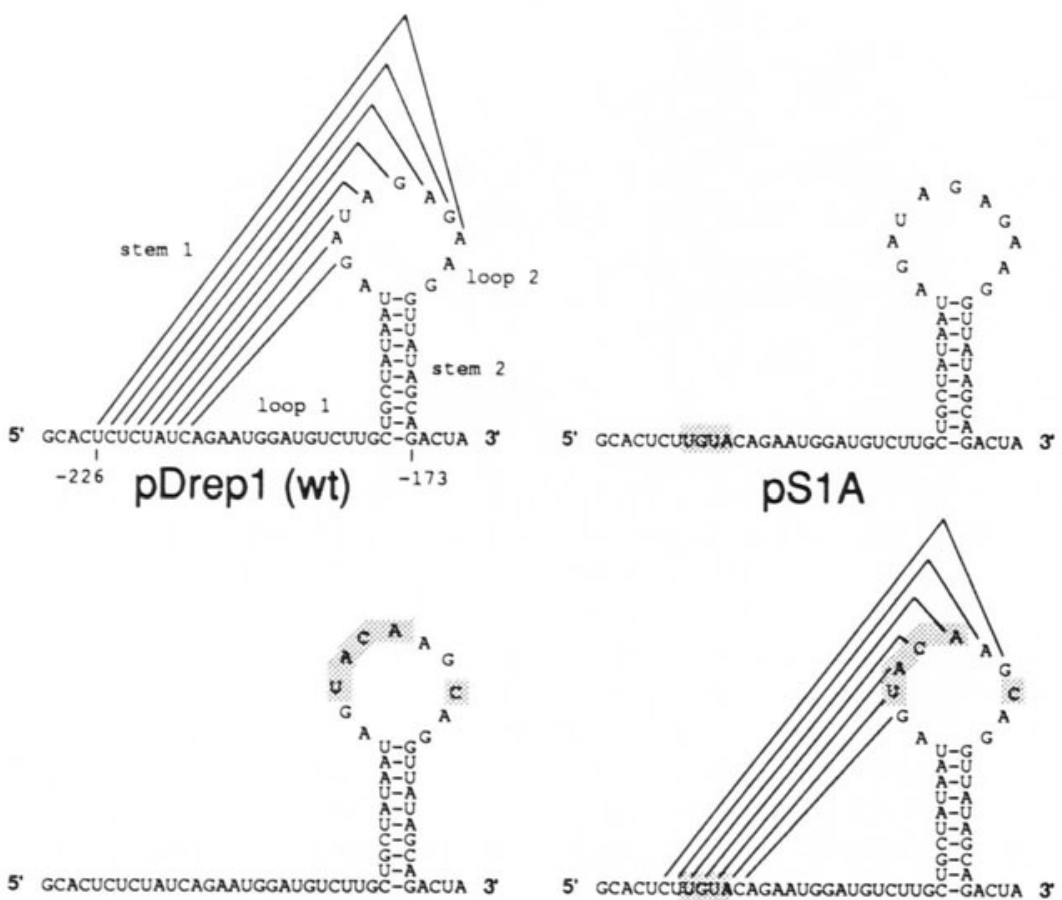

pS1B

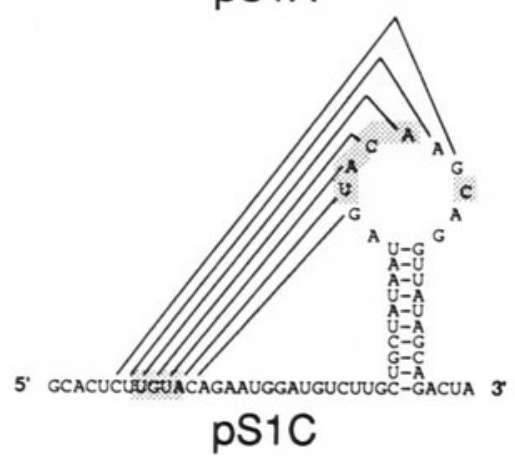

Figure 1. Proposed pseudoknot in the bovine coronavirus $3^{\prime}$ UTR and the mutations used to test its role in RNA replication A Wild-type pseudoknot in the genome 3' UTR It is shown here as part of the 3' UTR in the $22 \mathrm{~kb}$ reporter-containing defective interferıng RNA of BCV (pDrep1) B Mutant pS1A in which nucleotides CUAU in stem 1 were changed to UGUA C Mutant pSIB in which AUAGnnA in stem 1 were changed to UACAnnC D Mutant pS1C, the double mutant, in which the changes in pS1A and pS1B were combined to reform stem 1 which now has a stability of $-56 \mathrm{kcal} / \mathrm{mole}$ 


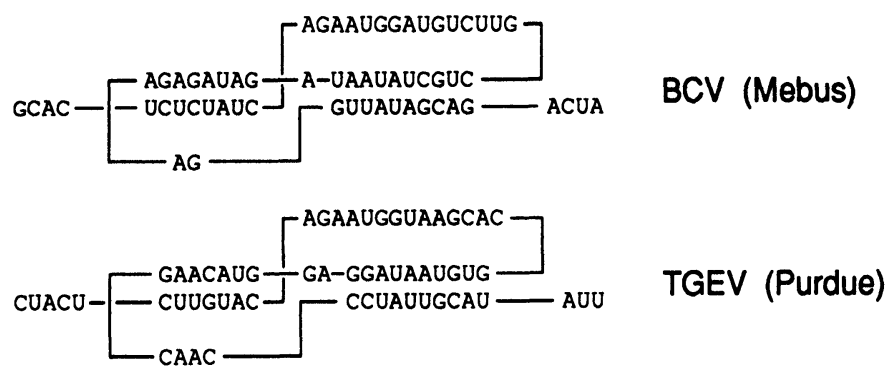

Figure 2. Comparison of the phylogenetically conserved pseudoknots between the bovine coronavirus and the porcine transmissible gastroenteritis coronavirus Stem 1 is shown on the left, and loop 1 is shown on the right Note the sequence divergence in the two stem regions

To determine whether the pseudoknotted structure is phylogenetically conserved among coronaviruses, the 3' UTR of the porcine transmissible gastroenteritıs virus (TGEV) and ten other mammalian coronaviruses was likewise examined Despite differences in nucleotide sequences, a pseudoknoted structure was found in the same relative position in TGEV (Fig 2) and the other coronaviruses examined (data not shown) Between BCV and TGEV, nucleotide similarities were $44 \%$ for stem 1 and $55 \%$ for stem 2 Yet in each case compensatory changes were found in TGEV that maintained stable stem structures These were predicted to be -58 and $-10 \mathrm{kcal} / \mathrm{mole}$ for stems 1 and 2, respectively A similar picture was found for the other coronaviruses

These observations led to the hypothesis that the pseudoknot must have evolved to serve a biological function To test the idea that it plays a role in RNA replication, mutations were made to disrupt the pseudoknot in pDrepl, and the effect of the mutations on replication of the DI RNA were tested $\mathrm{pS} 1 \mathrm{~A}$ and $\mathrm{pS1B}$ are constructs that have mutations on one side of stem 1 Point mutations were chosen to create mismatches predicted to destroy the thermodynamic stability of the stem The double mutant $\mathrm{pS1C}$ incorporates both sets of mutations and mimics the analogous stem in TGEV The three mutants were tested in a replication assay (Fig 3)

A Northern analysis showed that pDrepl with the wild-type pseudoknot replicated as evidenced by an increase in abundance and by having been passaged in virions (Fig 3, lanes 5, 6 and 7) Disruption of the stem in the single mutants pS1A and pS1B abolished replication when assessed by the same analysis (Fig 3, lanes 10,11 and 12, and 15, 16 and 17) On the other hand, the double mutant $\mathrm{pS} 1 \mathrm{C}$ with the compensated stem replicated at or near wild-type levels and became packaged into virions (Fig 3, lanes 20, 21 and 22) These experiments demonstrate the functional importance of this stem and the pseudoknot in RNA replication

\section{DISCUSSION}

To date, few phylogenetically conserved structural elements have been described in the $3^{\prime}$ untranslated region of coronaviruses that would suggest functional elements involved in RNA replication In addition to the pseudoknot reported here, an octameric consensus sequence GGAAGAGC is found approximately $70 \mathrm{nt}$ upstream from the poly (A) tail This conserved element, however, has not yet been shown to play a role in RNA synthesis

How the disrupted pseudoknot is preventing RNA replication in our studies is not known Since the pseudoknot and the octameric consensus sequence are in the $3^{\prime}$ UTR of genomic RNA, they might function as polymerase recognition or promoter sites for minusstrand RNA synthesis This, however, remains to be shown Both primary and secondary 


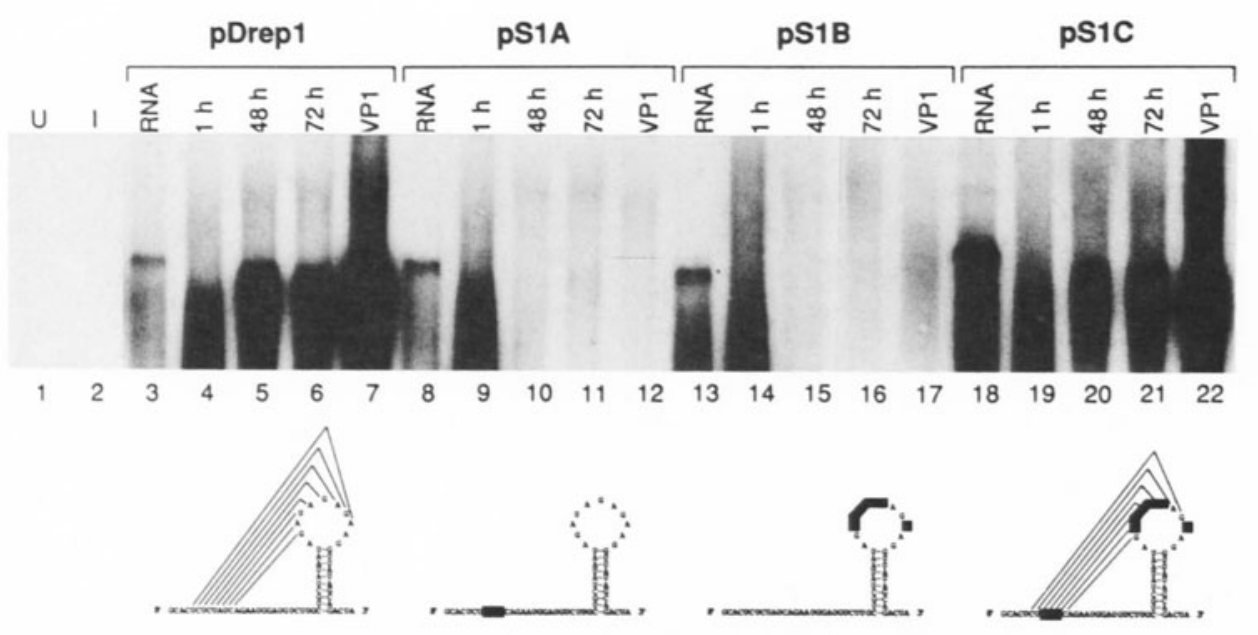

Figure 3. Importance of stem 1 in DI RNA replication. Synthetic transcripts of pDrepl and of the three mutants $\mathrm{pS1A}, \mathrm{pS1B}$, and pS1C were transfected into BCV helper virus-infected cells, and cytoplasmic RNA was extracted at various times posttransfection, or at $48 \mathrm{~h}$ after the first virus passage (VP1). Extracted RNA was separated by electrophoresis on a formaldehyde-agarose gel and probed in a Northern blot with radiolabeled probe for detection of the plus strand of the reporter sequence. Note the presence of the DI RNA in lanes identified pDrep 1 and $\mathrm{pSIC}$, but not in lanes identified as $\mathrm{pS} 1 \mathrm{~A}$ and $\mathrm{pS} 1 \mathrm{~B}$.

structures have been shown to be important for RNA-dependent RNA polymerase promoter activity $^{5}$. Another mechanistic possibility is that the disrupted pseudoknot may be preventing translation of the pDrepl open reading frame and secondarily preventing replication. A dependency on the open reading frame for pDrep1 replication has been shown ${ }^{1}$, and a strong preference for maintenance of the open reading frame in the replication of MHV DI RNAs has been demonstrated ${ }^{6,7}$. Precedent for a pseudoknot in the $3^{\prime}$ UTR of a plant virus being important for translation of the positive stranded genome has recently been reported ${ }^{8}$.

Further studies are needed to determine the mechanistic role of the pseudoknot in coronavirus RNA replication.

\section{REFERENCES}

1. Chang R.-Y., Hofmann M.A., Sethna P.B., Brian D.A. A cis-acting function for the coronavirus leader in defective interfering RNA replication. J Virol 1994;68:8223-8231.

2. Horton R.M., Cai Z., Ho S.N., Pease L.R. Gene splicing by overlap extension: tailor made genes using the polymerase chain reaction. BioTechnıques 1990;8:525-535.

3. Tinoco I., Borer P.N., Dengler B., Levine M.D., Uhlenbeck O.C., Crothers D.M., Gralla J. Improved estimation of secondary structure in ribonucleic acids. Nature (London) New Biol 1973;246:40-41.

4. ten Dam E., Pleij K., Draper D. Structural and functional aspects of RNA pseudoknots. Biochemistry 1992;31:1665-1676.

5. Dreher T.W., Hall T.C. Mutational analysis of the sequence and structural requirements in Brome Mosaic Virus RNA fro minus strand promoter activity. J Mol Biol 1988;201:31-40.

6. De Groot R.J., Van der Most R.G., Spaan W.J.M. The fitness of defective interfering murine coronavirus DI-a and its derivatives is described by nonsense and frameshift mutations. J Virol 1992;66:5898-5905.

7. Kim Y.-N., Lai M.M.C., Makino S. Generation and selection of coronavirus defective interfering RNA with large open reading frame by RNA recombination and possible editing. Virology 1993;194:244-253.

8. Leathers V., Tanguay R., Kobayashi M., Gallie D.R. A phylogenetically conserved sequence within viral 3' untranslated RNA pseudoknots regulates translation. Mol Cell Biol 1993;13:5331-5347. 